University of Wollongong

Research Online

Faculty of Engineering and Information

Faculty of Engineering and Information

Sciences - Papers: Part B

Sciences

2016

Indoor position tracking using received signal strength-based fingerprint context aware partitioning

Matthew D'Souza

University of Queensland, dsouza@itee.uq.edu.au

Brendan Schoots

University of Wollongong

Montserrat Ros

University of Wollongong, montse@uow.edu.au

Follow this and additional works at: https://ro.uow.edu.au/eispapers1

Part of the Engineering Commons, and the Science and Technology Studies Commons

Research Online is the open access institutional repository for the University of Wollongong. For further information contact the UOW Library: research-pubs@uow.edu.au 


\title{
Indoor position tracking using received signal strength-based fingerprint context aware partitioning
}

\author{
Abstract \\ Mobile indoor localisation has numerous uses for logistics and health applications. Current wireless \\ localisation systems experience reliability difficulties in indoor environments due to interference and also \\ require a large number of wireless access points to ensure position accuracy and resolution. Localisation \\ using wireless channel propagation characteristics, such as radio-frequency (RF) receives signal strength \\ are subject to wireless interference. The Fingerprint Context Aware Partitioning (FCAP) tracking model \\ used received RF signal strength fingerprinting, combined with context aware information about the user's \\ indoor environment. The authors show the use of context aware information in the FCAP model, reduces \\ the effect of wireless interference and lowers the spatial density of access points required. The wireless \\ localisation network consisted of reference nodes placed at locations in a building. Reference nodes are \\ used by mobile nodes, to localise a user's position. The authors tested the FCAP model in a typical indoor \\ environment and compared the performance and accuracy to other received signal strength indicator \\ fingerprint localisation methods. They found the FCAP model had improved performance and was able to \\ achieve a similar accuracy to other protocols, with fewer reference nodes.

\section{Disciplines} \\ Engineering | Science and Technology Studies

\section{Publication Details} \\ M. D'Souza, B. Schoots \& M. Ros, "Indoor position tracking using received signal strength-based \\ fingerprint context aware partitioning," IET Radar, Sonar and Navigation, vol. 10, (8) pp. 1347-1355, 2016.
}

This journal article is available at Research Online: https://ro.uow.edu.au/eispapers1/94 
$0000 ; 00: 1-16$

\title{
Indoor Position Tracking using Received Signal Strength based Fingerprint Context Aware Partitioning
}

\author{
Matthew D'Souza*, Brendan Schoots ${ }^{\dagger}$ and Montserrat Ros ${ }^{\dagger}$ \\ *School of Information Technology and Electrical Engineering, The University of Queensland, Brisbane, Australia \\ ${ }^{\dagger}$ School of Electrical, Computer and Telecommunications Engineering, University of Wollongong, Wollongong, Australia
}

\begin{abstract}
Mobile indoor localisation has numerous uses for logistics and health applications. Current wireless localisation systems experience reliability difficulties in indoor environments due to interference and also require a large number of wireless access points to ensure position accuracy and resolution. Localisation using wireless channel propagation characteristics, such as RF receive signal strength are subject to wireless interference. The Fingerprint Context Aware Partitioning (FCAP) tracking model used received RF signal strength fingerprinting, combined with context aware information about the user's indoor environment. We show the use of context aware information in the FCAP model, reduces the effect of wireless interference and lowers the spatial density of access points required. The wireless localisation network consisted of reference nodes placed at locations in a building. Reference nodes are used by mobile nodes, to localise a user?s position. We tested the FCAP model in a typical indoor environment and compared the performance and accuracy to other RSSI Fingerprint localisation methods. We found the FCAP model had improved performance was able to achieve a similar accuracy to other protocols, with fewer reference nodes.
\end{abstract}

KEYWORDS

2D Localisation; Wireless Sensor Network; Zigbee; RSSI Fingerprint, Propagation Channel Model

\section{INTRODUCTION}

Real-time position localisation of people is a widely sought after function in numerous applications for emergency services, location based services, social networking and sports and health domains. Commonly used real-time position tracking systems have become popular due to the availability of Global Positioning System (GPS). Indoor localisation tracking of people with unobtrusive, wearable sensors has valuable potential for applications where position tracking and motion activity monitoring is also useful. For example, providing a health-care application in the form of remote monitoring and tracking of aged-care patients can improve their safety and other care aspects.

We developed a Fingerprint Context Aware Partitioning (FCAP) tracking model for 2D tracking of people within a building. The FCAP model used a form of signal strength localisation, combined with a context information about the surrounding indoor environment, to improve localisation accuracy and reliability. Current localisation techniques depend on using sensing infrastructure already present in the environment such as visual markers, wireless LAN hotspots, cellular networks or GPS satellite coverage. However, indoor environments exhibit multi-path interference to Radio Frequency 
(RF) wireless technologies because of the presence of physical obstacles such as metal beams or walls. Hence this causes outdoor RF based localisation technologies such as GPS to function inaccurately indoors because of signal degradation. RF localisation methods such as Received Signal Strength Indicator (RSSI) or Time of Arrival also experience inaccuracies and reliability issues when operating indoors.

The FCAP tracking model used RSSI fingerprinting. Fingerprinting is a form of landmark localisation that uses distinct RSSI signatures to estimate a position. Current fingerprint base localisation methods such as [1, 2] typically require a high spatial density of wireless access points to ensure high position accuracy and resolution. The FCAP tracking model extends fingerprinting by using context-aware information, such as a building floorplan. The FCAP model incorporates context aware information to filter the RSSI fingerprints used for location estimation, in order to minimise location error. Context information such as the location of walls can be used to determine which RSSI fingerprints are nearby and not behind a wall. Distortions can be caused by RSSI fingerprints that are located behind a wall. Such RSSI fingerprints may appear to be closer or further away then in reality, when using traditional wireless range estimation techniques. The use of context aware information allowed the FCAP model to improve the reliability of the position accuracy, while depending on varying fingerprint granularity.

Our wireless localisation system used a low powered wireless sensor network infrastructure which consisted of reference nodes placed at predetermined coordinates in a building level. The reference nodes were used to determine the coordinates of the user within the region covered by the localisation network and the user carried a mobile node to determine their current position. We evaluated the FCAP tracking model in a typical and realistic indoor environment. We investigated the various performance aspects and advantages of the FCAP tracking model. We also compared the FCAP model to other common received signal strength based Fingerprint Localisation techniques.

\section{BACKGROUND LITERATURE}

Receive Signal Strength Indicators (RSSI) are used for indoor and outdoor localisation [3]. Common RSSI localisation techniques are RSSI fingerprinting, RSSI triangulation and trilateration. RSSI Fingerprinting identifies specific positions with RSSI values, while RSSI triangulation and trilateration associate RSSI with distance or angular trajectory between receiver and known transmitter positions, to localise [3, 4]. Hightower et al [5] used the Placelab geophysical location system based on RF fingerprinting with WLAN hotspots and GSM transmitters to determine a user's position. Localisation accuracy was found to be less then GPS, with 20-25m using wireless LAN and 100m to 150m for GSM broadcast towers.

Bahl et al [1] explored the use of RSSI Fingerprint Localisation with WLAN, using the RADAR algorithm. Youssef et al [2] conducted research into stochastic modelling based on the RADAR algorithm, which required extensive mapping of RF fingerprints. Youssef et al [2] reported the best case for the Horus (RADAR based) algorithm was $0.7 \mathrm{~m}-1.3 \mathrm{~m}$, with the worse case of $3 \mathrm{~m}$. However, the number of access points required a minimum of 6 for each RSSI fingerprint location. To get high accuracy, a high spatial density of access points is typically required. This is disadvantageous in indoor situations due to placement and power requirements of the access points.

Pahlavan et al [6] and Pourhomayoun et al [7] present the advantages of indoor localisation using WLAN with statistical modelling. Xiang et al present a form of context aware wireless WLAN localisation [8], which achieved an accuracy of $2 \mathrm{~m}$ for static objects and $5 \mathrm{~m}$ for moving objects. In [8], a state based learning approach was used, which required an extensive training phase. Compared to the FCAP model, a similar accuracy of 3m was achieved using 3 Access Points. Unlike the [8], no extensive training was required. Rasool et al present a statistical analysis of Gaussian range estimation for WSN [9]. They found that the goodness-of-fit statistical analysis of the experimental results suggest that range estimation error is not Gaussian distributed. Pourhomayoun et al [10] present an indoor localisation system based on spatial sparsity and a wireless sensor network. Chirakkal et al [11] used a smartphone based WLAN based RSSI Fingerprint localisation system and achieved 2.5m accuracy, indoors. Pahlavan et al [12], Hatami et al [13] and Yongguang et al [14] present classic RF localisation systems using TOA or signal strength. 


\section{INDOOR LOCALISATION NETWORK OVERVIEW}

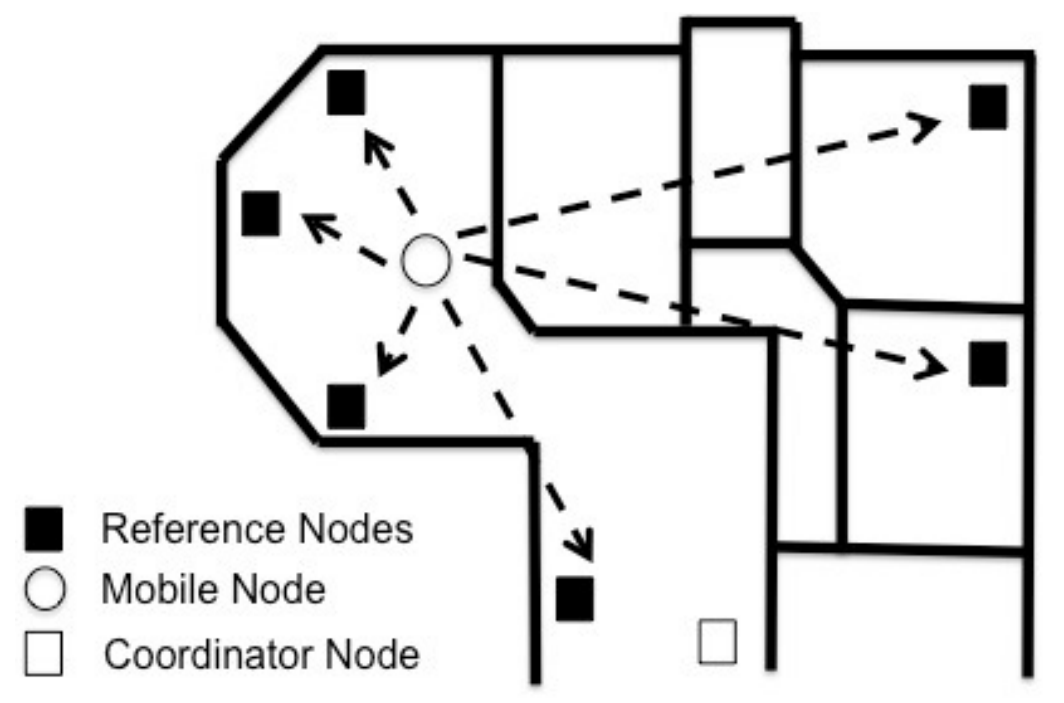

Figure 1. Overview of Wireless Sensor Network for RSSI Fingerprint Context Aware Partition Tracking Model

Wireless Network infrastructure used for both indoor and outdoor localisation, tends to be computationally intensive with high power consumption. Wireless sensor networks can be used for localisation but are also operate at low power. The ZigBee/802.15.4 [15] wireless communications protocol is used by the localisation network. Zigbee is a low data rate wireless communications protocol that can operate on devices with limited computing or power resources and cater for large networks of active devices [15].

The localisation network as seen in Figure 1 consisted of three types of nodes: coordinator, reference and mobile. Mobile nodes were carried by users to determine their current location. Reference nodes were used to determine a mobile node's position via multilateration. The server connected to the coordinator node displays the current positions of the mobile nodes on a building floorplan.

\subsection{Coordinator Node}

The coordinator node, seen in Figure 2, receives and visualises the location coordinates of each mobile node, using the server computer. The coordinator node communicates with the mobile node via the Zigbee mesh routing connection, using a CC2430 Zigbee/802.15.4 module [16]. The coordinator node is connected to a server computer by a serial connection. The server computer tracks the position of the mobile node using the context aware tracking process, as described in later sections. The coordinator node was powered by standard mains electricity.

\subsection{Reference Node}

The reference node, seen in Figure 3, communicates with the coordinator node via a Zigbee network connection. The position of each reference node is known by the coordinator node. The reference nodes are used by the mobile nodes for multilateration. The reference node used the CC2430 Zigbee/802.15.4 wireless transceiver module [16].

\subsection{Mobile Node}

The mobile node determines a user's position using RSSI. The mobile node detects reference nodes in near proximity and uses the RSSI to calculate its position. The predicted position is transmitted to the coordinator node via the reference node 


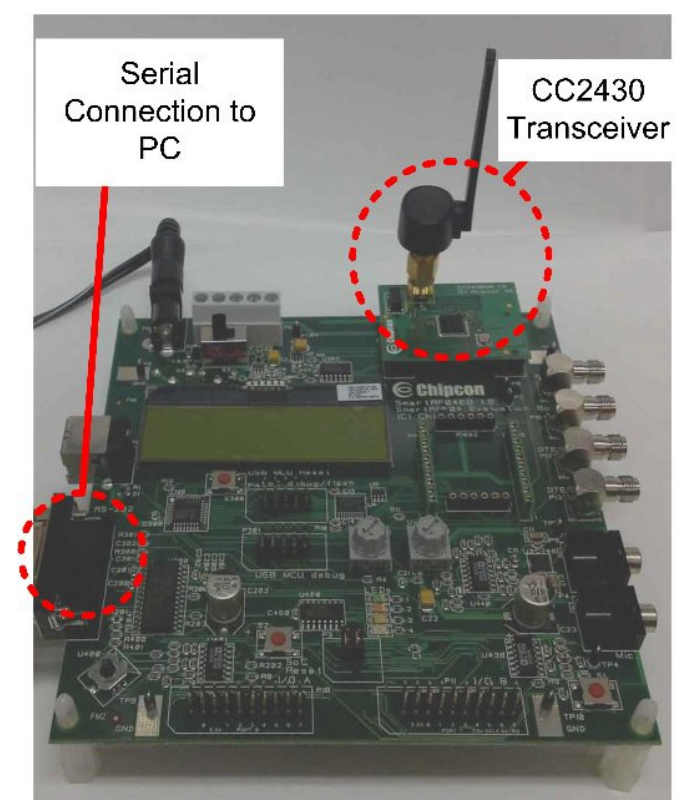

Figure 2. Coordinator Node Platform

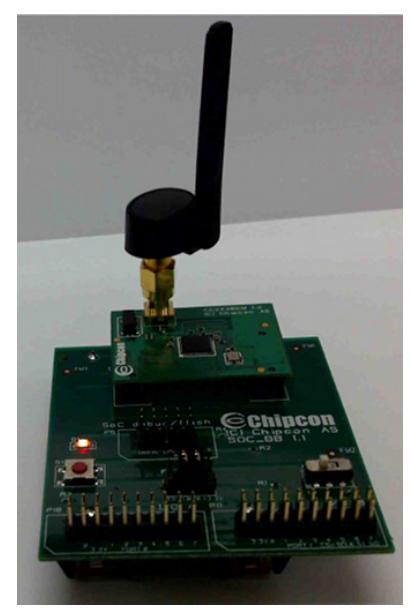

Figure 3. Reference and Mobile Node Platform

network. The mobile node used the CC2431 Zigbee/802.15.4 Location Engine Module [16]. The CC243 has an additional Location Engine Module to process RSSI in range calculations for localisation. The mobile node is powered by a battery, which allows it to be portable.

\subsection{Network Operation}

The range distances are approximated using RSSI and the coordinates of the reference nodes. Figure 4 shows how the mobile nodes interacted with the reference nodes. The mobile node periodically transmitted the RSSI measure messages to the nearest reference nodes in range (Figure 4A). The reference nodes use the RSSI measure messages to calculate the RSSI between a reference and mobile node. Five messages were used to calculate an averaged RSSI value. Using five RSSI values will ensure that fluctuations in the RSSI are minimised. The five messages were transmitted over a $2 \mathrm{~s}$ period (message sent every $400 \mathrm{~ms}$ ). We found this was sufficient to ensure that the RSSI value could be reliably measured and to calculate and display the mobile node's position, with minimal latency. 


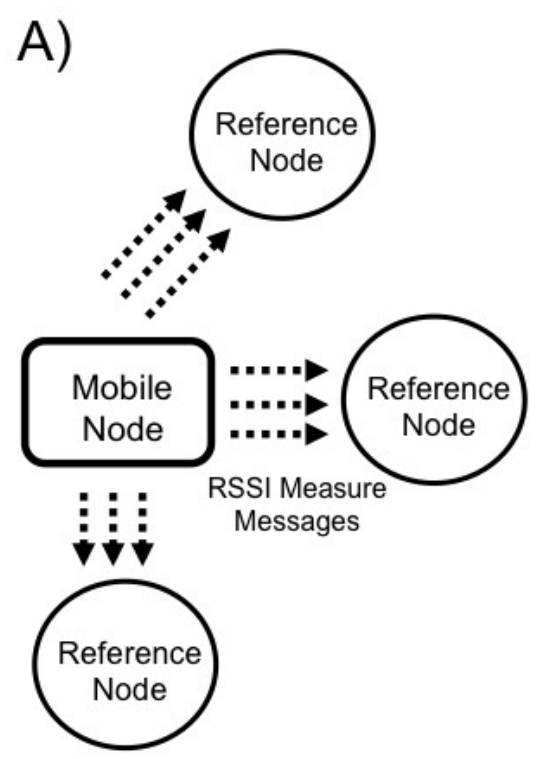

Mobile Node Sends Multiple RSSI Messages

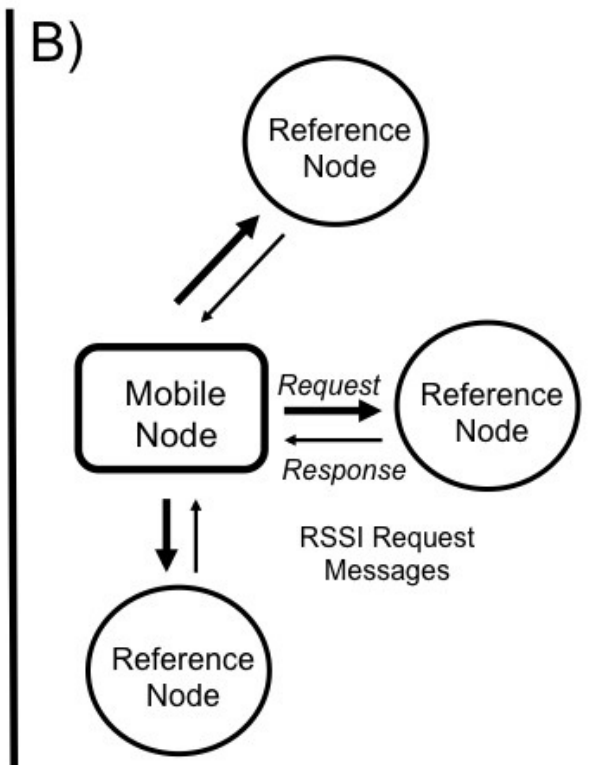

Mobile Node Requests RSSI of Reference Nodes

Figure 4. Mobile Node Receive Signal Strength Measuring Process

As seen in Figure 4B), once a series of RSSI Measure messages has been transmitted, the mobile node will then transmit an RSSI Request message to all reference nodes in range. Each reference node will then respond with its calculated RSSI values. These RSSI response messages are received by the coordinator node.

\section{FINGERPRINT CONTEXT-AWARE PARTITIONING TRACKING MODEL}

The Fingerprint Context Aware Partitioning (FCAP) tracking model estimates a receiver's position using RSSI fingerprinting. Fingerprints are a set of RSSI values, unique to specific locations. The use of fingerprints can determine the likely position of the mobile node by estimating which are the nearest reference nodes, using the mobile node's RSSI value. However conventional methods using fingerprints can be unreliable due to distortions of measuring RSSI caused by multipath and other forms of RF interference. The use of context-aware information for fingerprint partitioning enables a more reliable means of determining the nearest reference nodes.

Figure 5 shows an overview of the FCAP model. Table I describes each stage used in the FCAP model. The FCAP model consists of a number of stages used to filter and select the nearest reference nodes to the mobile node, based on the mobile node's RSSI value. The FCAP model uses the mobile node's RSSI values, RSSI fingerprint database and the building floorplan features. The FCAP model determines which RSSI fingerprints are nearest to the mobile node. Each FCAP sub sorting stage is used to select the nearest RSSI fingerprint to the mobile node, based on contextual information.

As mentioned previously in Section 3.4, the mobile node's RSSI value is averaged using five RSSI samples. First, the FCAP model estimates the likely position of the mobile node using circle partitioning. This estimate uses contextaware information provided by the floorplan and sub sorting of the RSSI values to refine the likely position. Finally, multilateration is used to estimated the position of the mobile node. 


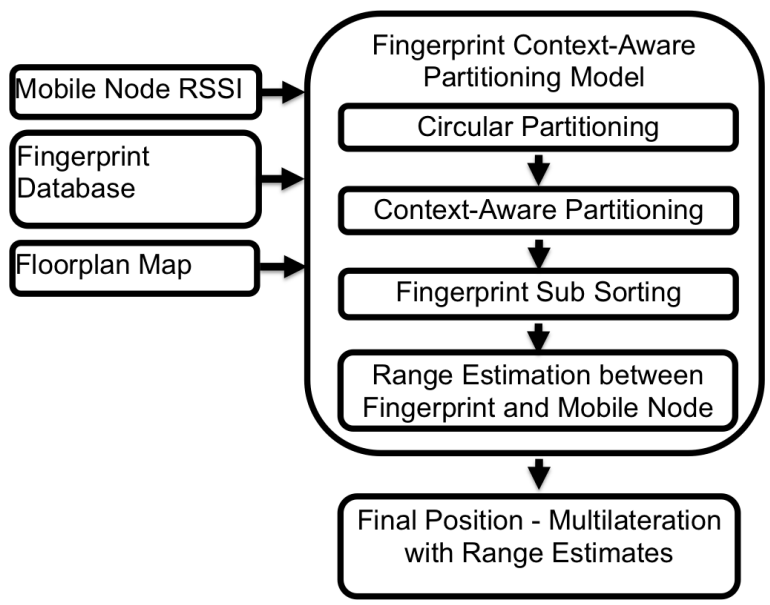

Figure 5. Stages of Fingerprint Context Aware Partitioning Tracking Model

\begin{tabular}{c|c}
\hline Stage & Description \\
\hline Mobile Node RSSI & Input of RSSI value extracted from 5 messages received \\
from Mobile Node.
\end{tabular}

Table I. FCAP Stages Description (From Figure 5)

\subsection{Circular Partitioning}

The circular partitioning process is used to determine the mobile node's nearest reference nodes within a certain radius. First, the reference node with the highest RSSI fingerprint is used as the centre of a circular area to partition the reference nodes that are within the valid area. The context-information of the building floorplan is then used to reduce the number of RSSI fingerprints that are within the valid area. An example of the circular partitioning process can be seen in Figure 6, where the bounded circle represents the partition encompassing a reference node (reference node 6 in Figure 6), and the actual mobile node's location. The valid fingerprint set can be reduced to the four fingerprints within the circular partition area.

The radius of the circular partition area determines the performance and accuracy of the subsequent localisation algorithms. For optimal performance, the selected radius should be as small as possible while still encompassing the mobile node's location. The radius must be defined such that it is equal to or greater than, the distance between the reference and mobile node. As the mobile node's location is unknown, the radius represents a best estimate of the maximum separation. 


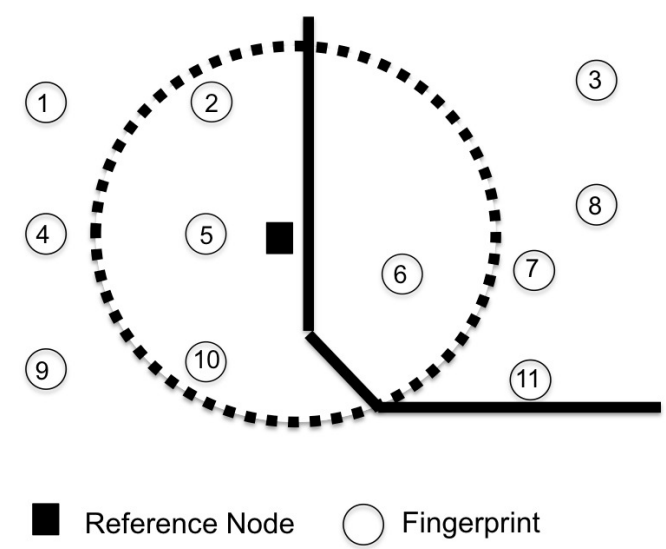

Figure 6. Circular Reference Node Partitioning Example

A logarithmic range radius algorithm was used to calculate the radius of the circular partitioning area by applying a fixed path loss logarithmic range. The radius or distance is calculated using the difference between reference node's RSSI and the absolute transmission power of the mobile node, for a fixed path loss exponent $n$, as shown in Equation 1 . The path loss exponent is used to represent the RF attenuation specific to an environment [17]. The path loss exponent is typically derived from experimental results. Table II shows the path loss exponents used test the FCAP model. The radius is set to the range calculated between the reference and mobile node. Logarithmic range was found to be well suited to partitioning as it is most accurate for high RSSI values from reference nodes within near proximity. A minimum radius of $2.5 \mathrm{~m}$ was used to prevent excessive exclusion of neighbouring fingerprints.

$$
d=10^{\frac{-R_{m}-A}{10 n}}
$$

Where $d$ is the radius distance around the mobile node, $R_{m}$ is the RSSI measurement value of the mobile node, $A$ is the absolute transmission power and $n$ is the path loss exponent.

\subsection{Context-Aware Partitioning}

Indoor environments can exhibit wireless interference due to the metallic structure present in the environment, multipath signal interference and other wireless devices. Such interference can distort received signal strength measurements, which will cause inaccuracies for RSSI fingerprint based localisation systems. In order to overcome wireless channel interference induced position errors, we developed a context-aware fingerprint sub sorting process to determine the likelihood of the RSSI fingerprints to be used. The context aware information used was the floorplan of the indoor environment, which consisted of a map with regions mapped with the probability of likely RSSI fingerprints available. The regions were dependent on the physical layout of the environment, such as walls. Position validity was approximated by detecting if the mobile node's track had to move through a wall or barrier, to its predicted position. Figure 6 shows an overview of the context-aware partitioning process used to check the validity of fingerprints to be used.

\subsection{Fingerprint Sub Sorting using Path Loss Exponents}

A fingerprint is a unique RSSI value measured at a particular set of coordinates. Fingerprint sub sorting is a process of selecting fingerprints for the multilateration position calculation, based on a path loss. The path loss exponent can be calculated for a fingerprint and a reference node. The path loss exponent represents the amount of RF attenuation or interference that can be experienced when measuring RSSI at a particular fingerprint's location. When selecting fingerprints based on an RSSI measurement of a signal from a reference node, a situation can occur where several fingerprints have similar RSSI values. Due to RF signal attenuation caused by the multi path interference of the surrounding environment, 
fingerprints with similar RSSI value differences may not be equidistant from the reference node. Therefore we sub sorted the RSSI fingerprints were based on their path loss exponent, calculated using the RSSI between the reference node and fingerprint location (equation 1). Preference is given to selecting fingerprints which experience lower attenuation.

\subsection{Multilateration Position Calculation}

Once the fingerprints have been determined, multilateration is used to calculate the mobile node's position. As seen in Equation 2, the multilateration position calculation process used the known positions of the selected reference nodes and corresponding RSSI values to determine the mobile node's position.

$$
\left[\begin{array}{c}
\left(x_{1}-x\right)^{2}+\left(y_{1}-y\right)^{2} \\
\left(x_{2}-x\right)^{2}+\left(y_{2}-y\right)^{2} \\
\cdots \\
\cdot \\
\left(x_{N_{r}}-x\right)^{2}+\left(y_{N_{r}}-y\right)^{2}
\end{array}\right]=\left[\begin{array}{c}
d_{1}^{2} \\
d_{2}^{2} \\
\cdots \\
\cdots \\
d_{N_{r}}^{2}
\end{array}\right]
$$

Where $x_{N} r$ and $y_{N} R$ represent the Cartesian coordinates of the reference nodes, $x$ and $y$ represent the Cartesian coordinates for the mobile node and $d_{N} R$ represents the distance between the reference and mobile nodes.

\subsection{Fingerprint Collection}

RSSI values were collected at various distinct measuring locations in the indoor test environment. These RSSI value vectors were placed into a database and were used to provide a unique fingerprint of each location. In some cases, fingerprint locations were constrained by the presence of immovable obstacles within the environment. The RSSI of all reference nodes within range was recorded at each fingerprint location. A minimum of three fingerprints were collected for each room, with a spacing of $1 \mathrm{~m}$ typically maintained between measurements.

Figures 7(a), 7(b), 7(c) and 7(d) display the RSSI fingerprint heat map for corresponding reference nodes. The measuring locations used are shown as crosses. The RSSI fingerprint heatmaps can vary for each reference node, due to its location and proximity to wall enclosures. The reference node locations are discussed in the next section. The coloured circle at each fingerprint represents RSSI value from the reference node at that point, with deep blue through to deep red representing a range of $-40 \mathrm{dBm}$ to $-90 \mathrm{dBm}$.

\section{EVALUATION}

The FCAP tracking model was tested in an indoor environment shown in Figure 8. The density placement of the reference nodes can affect the localisation accuracy using RSSI fingerprints. We tested the FCAP model by having a user walk a known path whilst carrying a mobile node. The walking path had 14 test points which were chosen to ensure that atleast 3 reference nodes were within range of the mobile node, due to the confined space of the test indoor environment. The walking path chosen also ensure that the user passed through as many rooms as possible, in order to maximise testing of the FCAP model. The test indoor environment was a confined space, which consisted of seven enclosed room areas, enclosing an area of $132 \mathrm{~m}^{2}$. The largest room area was $48 \mathrm{~m}^{2}$. The smallest room area was $16 \mathrm{~m}^{2}$. There were five intersecting internal walls throughout the test area. The test indoor environment was also chosen because it exhibited typical conditions encountered in normal urban dwellings, like internal walls. Testing was carried out for two distinct reference node layouts. Both of these layouts utilised, at most, one reference node per room. This was done due to the confined nature of the testing space. 


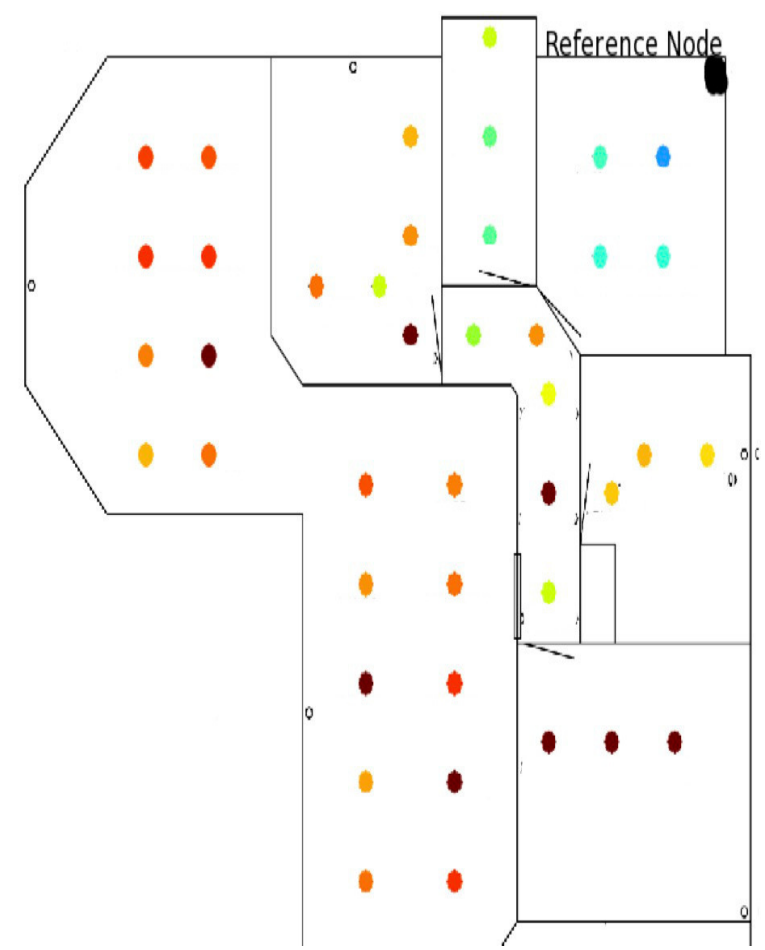

(a) Reference Node 1

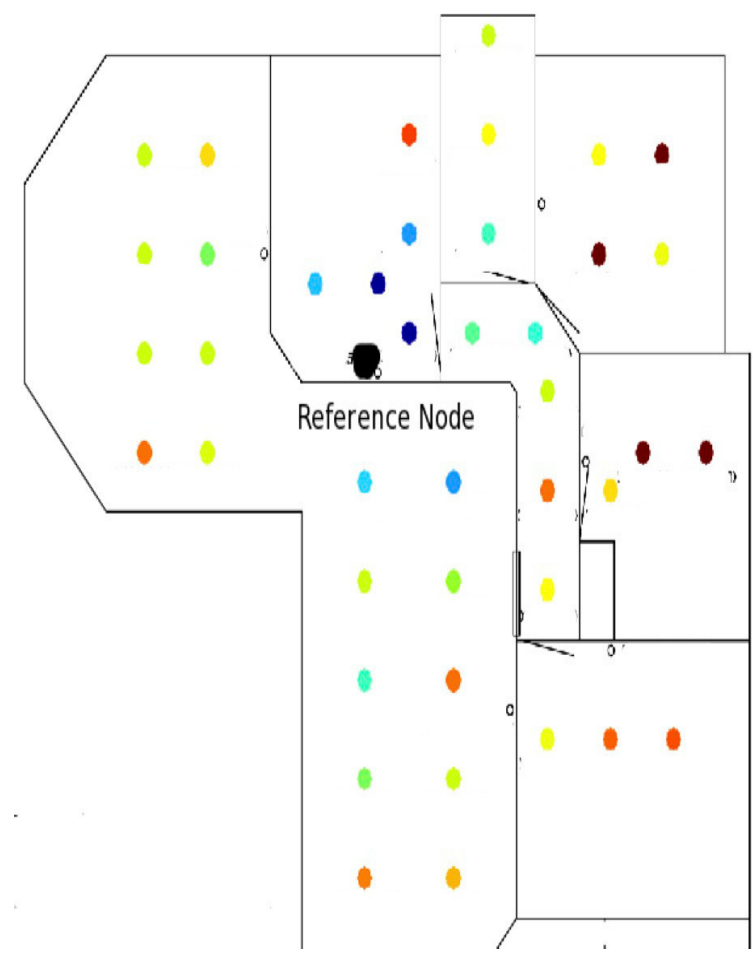

(c) Reference Node 3

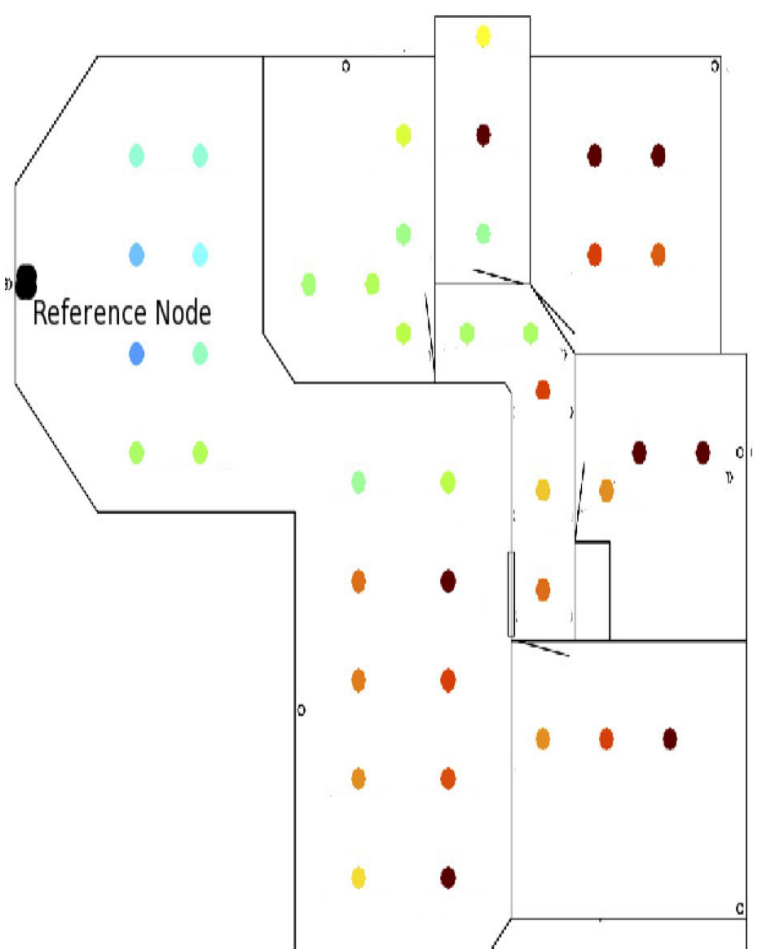

(b) Reference Node 2

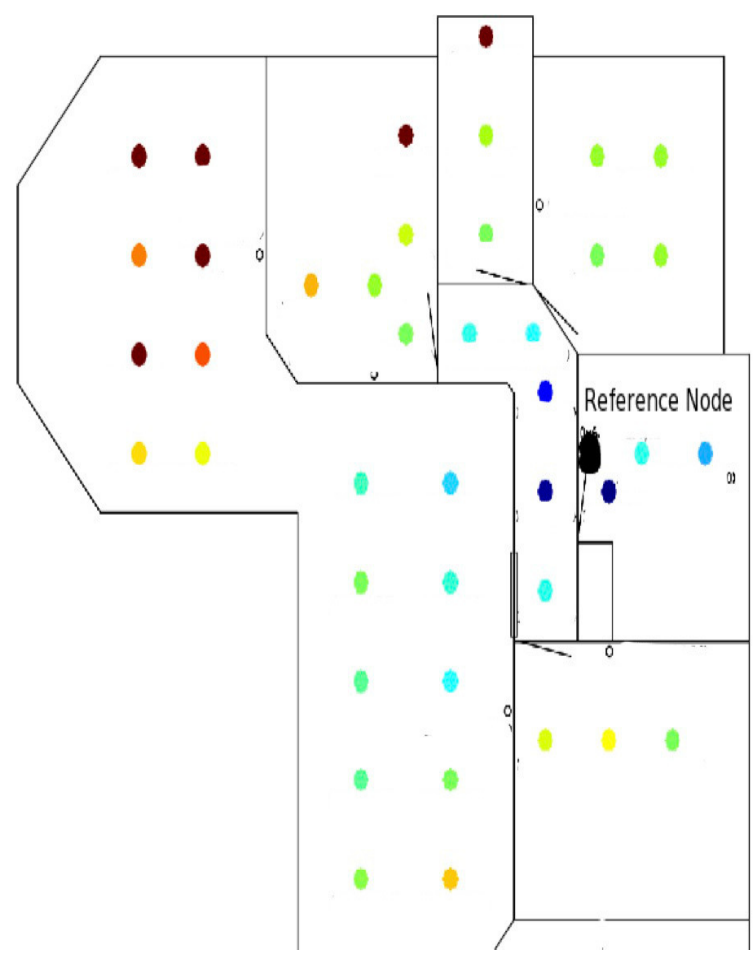

(d) Reference Node 4

Figure 7. RSSI Fingerprint Heat Maps 


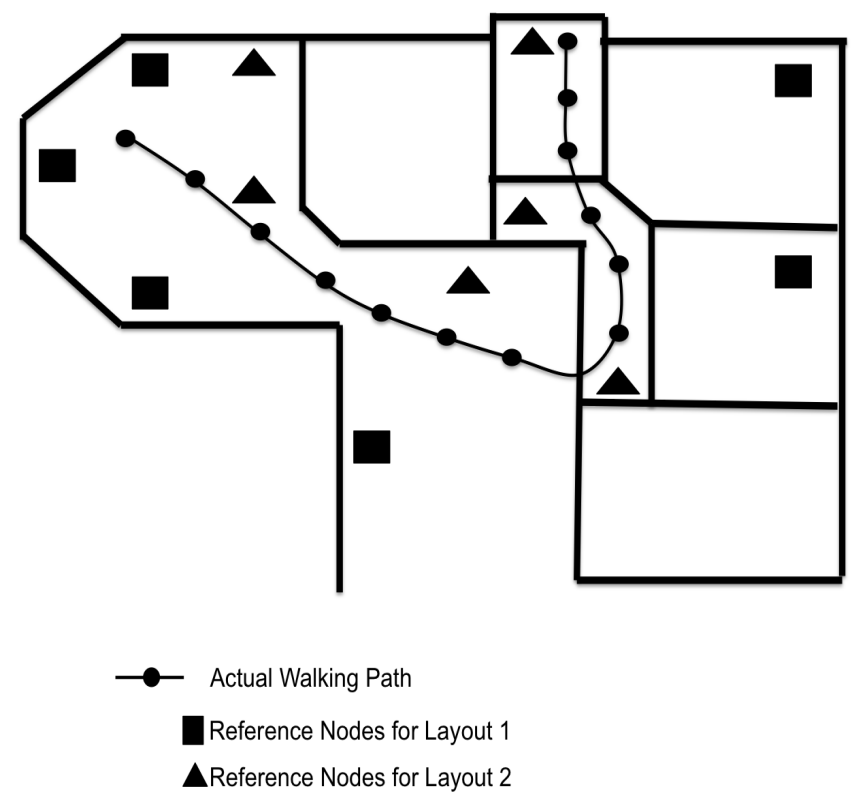

Figure 8. Walking Path and Reference Node Placements

The reference node locations are shown in Figure 8 for Layouts 1 and 2. Both layouts used the same number of reference nodes. Layout 2 had a more dense placement (smaller separation) of reference nodes, compared to Layout 1 . Layout 1 places reference nodes adjacent to perimeter walls enclosing the test area with the geometry of the reference nodes. Layout 2 places reference nodes adjacent to interior walls. Compared with Layout 1, this decreases separation between reference nodes toward the centre of the building, at the expense of greater separation at its extremities. Layout 2 was designed to test which room the mobile node is in and where the measured RSSI may be similar on either side of a wall. The path loss exponents for both layouts is shown in Table II. The path loss exponent can be used to represent the amount of wireless interference present in an environment. Due to Layout 1's smaller path loss exponent, Layout 1 exhibits less interference than Layout 2. For both layouts, we fixed the height of the reference and mobile nodes to be $1 \mathrm{~m}$ above the floor. We found this was suitable for the external antenna of the reference and mobile nodes used.

\begin{tabular}{lrr}
\hline Reference Node Layout & 1 & 2 \\
\hline Average Path Loss Exponent (n) & 3.16 & 4.48 \\
Standard Deviation & 2.44 & 1.58 \\
\hline
\end{tabular}

Table II. Path Loss Exponents for Layout 1 and 2

RSSI fingerprint measurements were collected for a typical path through the test environment. A total of 41 RSSI fingerprints was measured and averaged for each layout. The spacing between RSSI fingerprints was $1 \mathrm{~m}$. At least 5 measurements were averaged for each RSSI fingerprint. As shown in Figure 8, the path traversed rooms with and without reference nodes. RSSI readings was recorded at 14 different locations along the path, each approximately $1 \mathrm{~m}$ apart. Thirty repetitions of traversing the path for each layout, was conducted. Mobile node orientation matched the direction of travel at each point, which may differ from that used during fingerprint collection.

Both reference node layouts provide different node separation and geometry at the walking path measurement locations. It should be noted that Layout 2 provided significantly lower average distances between the reference nodes and measurement locations than Layout 1 . The walking paths average distance from the closest reference node was $2.75 \mathrm{~m}$ for Layout 1 and $1.33 \mathrm{~m}$ for Layout 2, while the average separation from all reference nodes was $5.73 \mathrm{~m}$ and $3.68 \mathrm{~m}$ for Layouts 1 and 2 respectively. 
We evaluated the performance of the FCAP with respect to accumulated position error. We also analysed the impact of variations of using different circular partitioning and sorting algorithms with the FCAP model. Our analysis of the performance of the FCAP was structured as the following:

- Comparison of FCAP with Multilateration, Least Squares, Correlation and KNN.

- Performance of radius calculation algorithms.

- Performance and comparison of different fingerprint sorting methods.

\section{COMPARISON}

We compared the use of the FCAP to other common RSSI Fingerprint localisation methods such as multilateration, least squares, correlation and KNN. Table III and IV shows the average error and standard deviation measured for layout 1 and 2, respectively. Figure 8 shows the walking path track used for testing. Figures 9(a), 9(b), 9(c) and 9(d) show the corresponding walking path track localisation using FCAP, multilateration, correlation and KNN. Figure 9(a) shows the localisation for layouts 1 and 2, using only the FCAP model. Figure 9(b) shows the localisation for layouts 1 and 2, using only multilateration. Figure 9(c) shows the localisation for layouts 1 and 2, using only correlation. Figure 9(d) shows the localisation for layouts 1 and 2, using only KNN. The average error was similar for multilateration, least squares and the FCAP model for Layout 1, as seen in Figures 9(b), 9(c) and 9(a). For Layout 2, the FCAP model had less error than that measured for multilateration and least squares with Layout 2.

\begin{tabular}{lrr}
\hline Layout & Avg. Error $(\mathrm{m})$ & Stand. Deviation $(\mathrm{m})$ \\
\hline FCAP & 3.16 & 2.1 \\
Multilateration & 2.57 & 1.62 \\
Least Squares & 2.44 & 1.58 \\
Correlation & 2.88 & 1.77 \\
KNN & 3.38 & 2.30 \\
\hline
\end{tabular}

Table III. Average Error and Standard Deviation for Layout 1

\begin{tabular}{lrr}
\hline Layout & Avg. Error $(\mathrm{m})$ & Stand. Deviation $(\mathrm{m})$ \\
\hline FCAP & 4.48 & 4.96 \\
Multilateration & 14.29 & 24.54 \\
Least Squares & 13.28 & 24.75 \\
Correlation & 2.12 & 1.43 \\
KNN & 3.09 & 2.85 \\
\hline
\end{tabular}

Table IV. Average Error and Standard Deviation for Layout 2

For layout 1, error results for the correlation and $\mathrm{KNN}$ based methods were similar compared to the FCAP model. For layout 2, the average position error and standard deviation for the correlation and KNN based methods were lower compared to the FCAP model or multilateration. The FCAP model's use of multilateration for the final position calculation would also contribute to a higher error than compared to $\mathrm{KNN}$ or correlation.

\subsection{FCAP Performance Evaluation}

The performance of different radius and sub sorting algorithmic implementations were evaluated for the FCAP model. The presence of the mobile node within a specific radius of the reference nodes was used as a performance indicator. The performance of the logarithmic radius calculation process was investigated and two RSSI sub sorting methods based on separation distance between reference and mobile nodes is also presented in this section. 


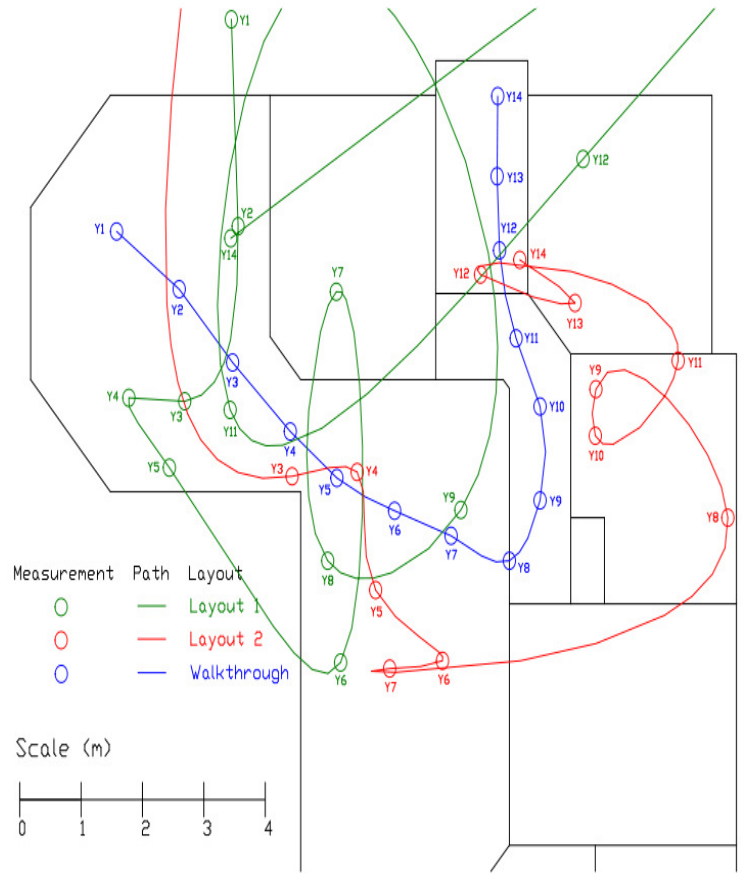

(a) FCAP

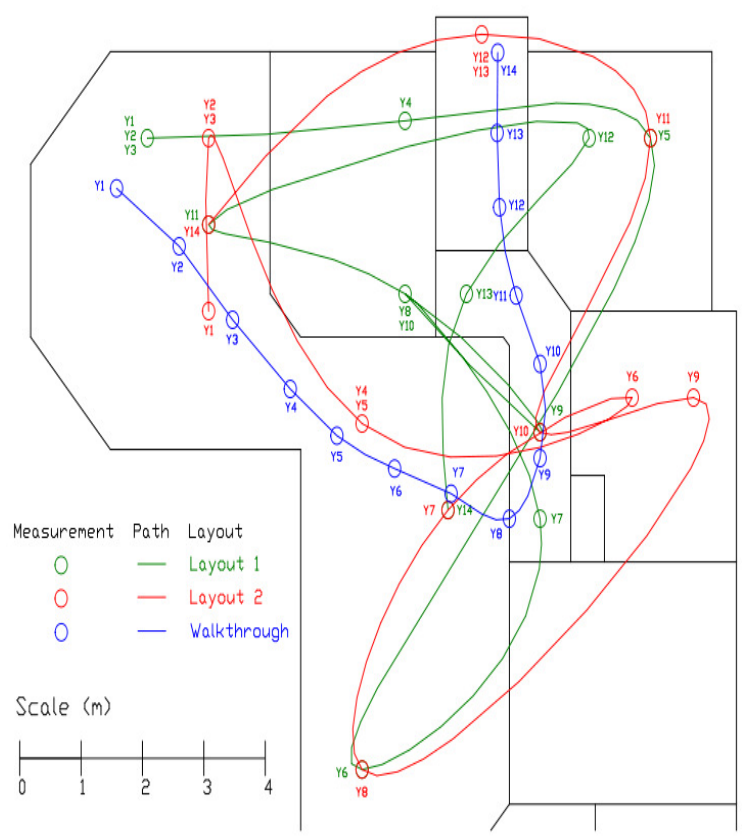

(c) Correlation

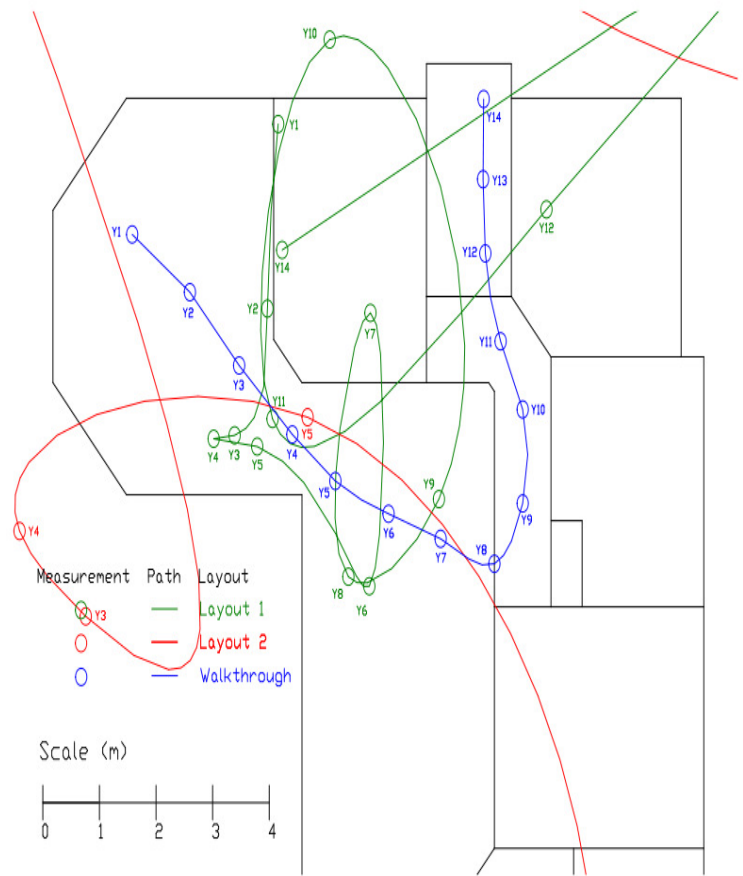

(b) Multilateration

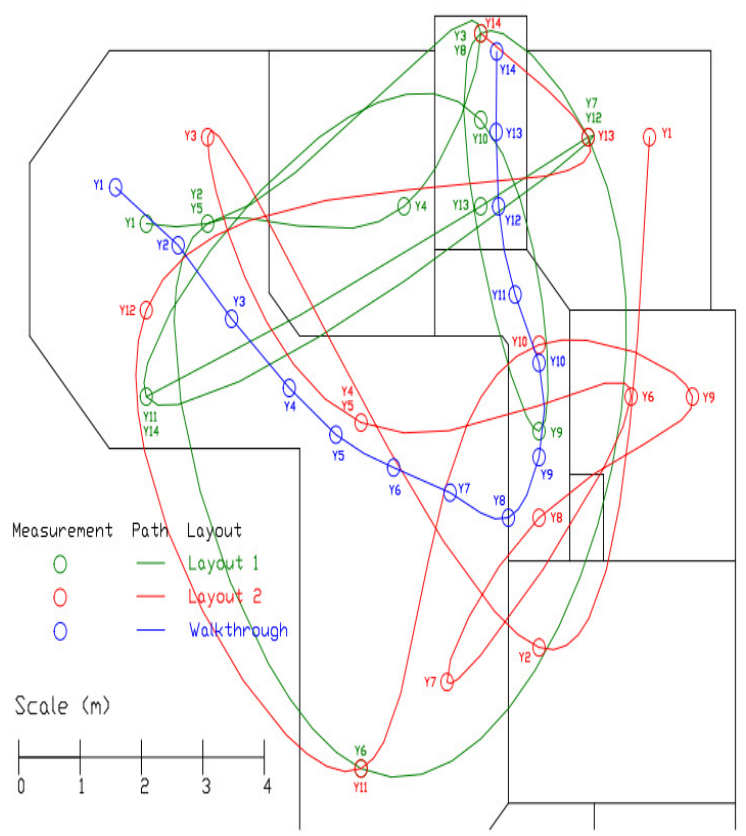

(d) $\mathrm{KNN}$

Figure 9. Walking Path Track Localisation Evaluation

\subsubsection{FCAP Radius Calculation Algorithm Performance}

The performance of the logarithmic range partitioning with two different layouts is shown in Table V. The average separation of walkthrough measurement locations from the reference nodes with highest RSSI was $3.87 \mathrm{~m}$ and $1.44 \mathrm{~m}$ for 


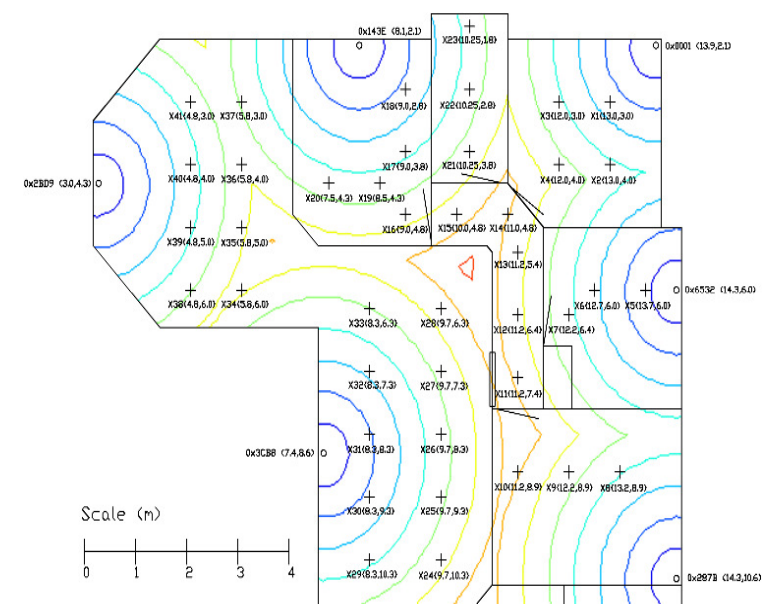

(a) Layout 1

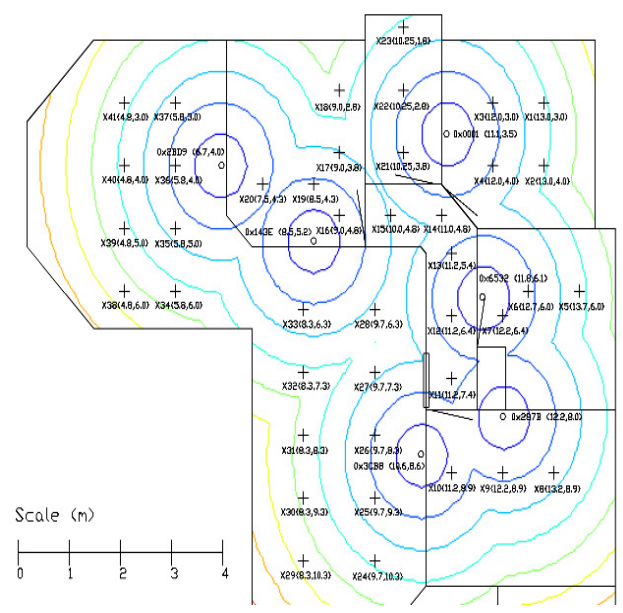

(b) Layout 2

Figure 10. Distance to Reference Node Heatmap

Layouts 1 and 2 respectively. As RSSI is dependent on distance from the reference node, the reduced mobile node presence of Layout 1 can be attributed to its higher average node separation. Logarithmic range was allowed the FCAP model to be more accurate when the reference node density was high.

\begin{tabular}{lrr}
\hline \multicolumn{1}{c}{ Reference Node Layout } & \multicolumn{1}{c}{1} & \multicolumn{1}{c}{2} \\
\hline Average Radius (m) & 3.94 & 3.07 \\
Average Fingerprints in Radius & 12.86 & 7.50 \\
Average as \% of Total Fingerprints & 31.36 & 18.29 \\
Mobile Node Presence (\%) & 64.29 & 100.00 \\
\hline
\end{tabular}

Table V. Logarithmic Range Radius Results

\subsection{FCAP Sub Sorting Performance}

The FCAP model used a path loss based sub sorting method. The performance results can be seen in Table VI. The performance of other methods such as the Distance to Reference Node and Average Distance Sub Sorting were compared to the path loss sub sorting method. The presence of the mobile node within the $5.6 \mathrm{~m}$ and $3.63 \mathrm{~m}$ radius of included reference nodes was high for all tested cases.

\begin{tabular}{lrr}
\hline \multicolumn{1}{c}{ Reference Node Layout } & \multicolumn{1}{c}{1} & \multicolumn{1}{c}{2} \\
\hline Average Radius (m) & 5.60 & 3.63 \\
Average Fingerprints in Radius & 21.00 & 19.57 \\
Average as \% of Total Fingerprints & 51.22 & 47.74 \\
Mobile Node Presence (\%) & 92.86 & 92.86 \\
\hline
\end{tabular}

Table VI. Path Loss Sub Sorting Results

\subsubsection{Distance to Reference Node Sub Sorting}

The Distance to Reference Node sub sorting method is used to sort fingerprints with respect to its distance from the selected reference node. Figure 10(a) and 10(b) show a heatmap of the distance to reference node for layouts 1 and 2. Preference was given to fingerprints with greater separation from the reference node. Selecting the most distant fingerprint 
increased the partition radius but also improve the position accuracy of the mobile node. However, this increased the size of the valid fingerprints set compared to the FCAP path loss based sub sorting method. The drawback of a larger set of valid fingerprints is the increased computational overhead in calculating the mobile node's position compared to the FCAP path loss based sub sorting method. As seen in Table VII, the position accuracy for both layouts was similar to that of the FCAP path loss based sub sorting method.

\begin{tabular}{lrr}
\hline \multicolumn{1}{c}{ Reference Node Layout } & \multicolumn{1}{c}{1} & \multicolumn{1}{c}{2} \\
\hline Average Radius (m) & 5.60 & 3.63 \\
Average Fingerprints in Radius & 21.00 & 19.57 \\
Average \% of Total Fingerprints & 51.22 & 47.74 \\
Mobile Node Presence (\%) & 92.86 & 92.86 \\
\hline
\end{tabular}

Table VII. FCAP Distance to Reference Node Sub Sorting Results

The mobile node's presence was equal to that of path loss sub sorting. Radius results were also near identical to those provided by path loss sub sorting. However, sub sorting by reference node distance is less efficient than by path loss, as it requires a continuous distance calculation. By contrast, fingerprint path loss values are calculated once.

\subsubsection{FCAP Average Sub Sorting Distance}

The average distance based sub sorting method used the average separation distance from the reference node, to sort the fingerprints. Average sub sorting distance results are shown in Table VIII. The presence of the mobile node was similar to that of the path loss and reference node distance algorithms. Decreases in the valid fingerprint set over the other sub sorting techniques were modest, even with the negligible decrease in the valid fingerprint set, and slight decrease of the percentage presence of the mobile node.

\begin{tabular}{lrr}
\hline \multicolumn{1}{c}{ Reference Node Layout } & \multicolumn{1}{c}{1} & \multicolumn{1}{c}{2} \\
\hline Average Radius (m) & 5.39 & 3.58 \\
Average Fingerprints in Radius & 19.79 & 19.21 \\
Average \% of Total Fingerprints & 48.26 & 46.86 \\
Mobile Node Presence (\%) & 85.71 & 92.86 \\
\hline
\end{tabular}

Table VIII. FCAP Average Distance Sub Sorting Results

The path loss sub sorting provided greater mobile node presence and consistency across both tested layouts than logarithmic range radius. However, reduction of the valid fingerprint set was not as great, decreasing the performance of subsequent localisation algorithms. The path loss sub sorting provides a balance between accuracy and performance in situations where the reference node density is unknown.

\section{CONCLUSION AND FURTHER WORK}

We presented a wireless indoor localisation system that 2D tracked users in an indoor environment, using the FCAP model. The indoor localisation system used a Zigbee based wireless sensor network. The network consisted of three nodes: coordinator, reference and mobile nodes. The mobile node's RSSI value with respect to the reference node was used by the FCAP model as an RSSI fingerprint. The FCAP model combined the mobile node's RSSI fingerprinting with context-aware information (building floorplan). Through the use of context aware information the FCAP model improved position accuracy of the mobile node.

We evaluated and compared the FCAP model to other RSSI fingerprint based localisation methods: multilateration, least squares, correlation and KNN. The FCAP model had a smaller position error when compared to multilateration and least 
squares, where the position of the mobile node was not restricted to areas geometrically covered by multiple reference nodes. This highlighted the advantage of the FCAP model as it allowed the unrestricted placement of the reference nodes.

The performance of the FCAP model was also investigated. The performance of the different radius calculation and RSSI fingerprint sub sorting methods were evaluated. It was found that the logarithmic radius calculation based process was found to be most effective in position accuracy by selecting significant RSSI values from reference nodes to be used by the FCAP model. Using path loss between the reference and mobile nodes, to sub sort the RSSI fingerprints was also found to be more effective when compared to using the separation distance between the reference and mobile nodes. One of the drawbacks of RSSI localization is the need to deploy a high density access points or reference nodes in order to localisation with high accuracy. This is disadvantageous in most indoor situations due to placement and power requirements.

Further work will involve investigating how mobile nodes can use cooperative communications with the FCAP model, to better improve position accuracy. Further work will include investigating how Wifi modules can be used to implement the indoor localisation network of reference and mobile nodes. Further work will also investigate the effects of the radius on the sensitivity of the FCAP performance.

\section{REFERENCES}

1. Bahl P, Padmanabhan V. RADAR: An In-Building RF-based User Location and Tracking System. INFOCOM 2000. Nineteenth Annual Joint Conference of the IEEE Computer and Communications Societies, vol. 2, 2000; 775-784 vol.2.

2. Youssef M and Agrawala A. The Horus WLAN Location Determination System. In Communication Networks and Distributed Systems Modeling and Simulation Conference, 2005; 205-218.

3. Seco F, Jimenez A, Prieto C, Roa J, Koutsou K. A Survey of Mathematical Methods for Indoor Localization. WISP 2009. IEEE International Symposium on Intelligent Signal Processing, 2009, 2009; 9 -14.

4. Liu H, Darabi H, Banerjee P, Liu J. Survey of Wireless Indoor Positioning Techniques and Systems. IEEE Transactions on Systems, Man, and Cybernetics, Part C: Applications and Reviews November 2007; 37(6):1067 -1080 .

5. Hightower J, LaMarca A, Smith I. Practical Lessons from Place Lab. Pervasive Computing, IEEE July 2006; 5(3):32 -39 .

6. Pahlavan K, Krishnamurthy P, Beneat J. Wideband radio propagation modeling for indoor geolocation applications. Communications Magazine, IEEE Apr 1998; 36(4):60-65.

7. Pourhomayoun M, Jin Z, Fowler ML. Indoor localization, tracking and fall detection for assistive healthcare based on spatial sparsity and wireless sensor network. Int. J. Monit. Surveill. Technol. Res. Apr 2013; 1(2):72-83. URL http://dx.doi.org/10.4018/ijmstr.2013040105.

8. Xiang Z, Song S, Chen J, Wang H, Huang J, Gao X. A wireless lan-based indoor positioning technology. IBM Journal of Research and Development Sep 2004; 48(5.6):617-626.

9. Rasool I, Kemp A. Statistical analysis of wireless sensor network gaussian range estimation errors. Wireless Sensor Systems, IET March 2013; 3(1):57-68.

10. Pourhomayoun M, Fowler M. Improving wlan-based indoor mobile positioning using sparsity. Signals, Systems and Computers (ASILOMAR), 2012 Conference Record of the Forty Sixth Asilomar Conference on, 2012; 1393-1396.

11. Chirakkal V, Park M, Han DS. Navigating through dynamic indoor environments using wifi for smartphones. Consumer Electronics Berlin (ICCE-Berlin), 2014 IEEE Fourth International Conference on, 2014; 376-378.

12. Pahlavan K, Li X, Makela JP. Indoor geolocation science and technology. Communications Magazine, IEEE Feb 2002; 40(2):112-118. 
13. Hatami A, Pahlavan K, Heidari M, Akgul F. On rss and toa based indoor geolocation - a comparative performance evaluation. Wireless Communications and Networking Conference, 2006. WCNC 2006. IEEE, vol. 4, 2006; 22672272.

14. Chen Y, Kobayashi H. Signal strength based indoor geolocation. Communications, 2002. ICC 2002. IEEE International Conference on, vol. 1, 2002; 436-439.

15. ZigBee Alliance. ZigBee Specification. "www.zigbee.org/en/specdownload/" 2006.

16. Texas Instruments Inc. Texas Instruments CC2431 ZigBee Development Kit 2007.

17. Stuber GL. Principles of Mobile Communication. 2 edn., Kluwer Academic Publishers, 2002. 